International Journal of Biomedicine I June 2019 - Volume 9, Issue Suppl_1: Abstracts From the Second Russian International Conference "Cryo-electron microscopy 2019: achievements and prospects"

\author{
POSTER ABSTRACT PRESENTATIONS
}

SESSION TITLE: STRUCTURE AND FUNCTIONS OF THE TRANSCRIPTION AND TRANSLATION APPARATUS OF THE CELL

DOI: 10.21103/IJBM.9.Suppl_1.P18

\title{
Abstract P-18: PARP1-Induced Structural Rearrangements are Enhanced in Cisplatin-Treated Nucleosomes
}

\author{
Natalya Maluchenko $^{1}$, Alexey Feofanov ${ }^{1,2}$, Mikhail Kirpichnikov ${ }^{1,2}$, Vasily Studitsky ${ }^{1,3}$, \\ Nadezhda Gerasimova ${ }^{1}$
}

${ }^{1}$ Faculty of Biology, Lomonosov Moscow State University, Moscow, Russia; ${ }^{2}$ Shemyakin-Ovchinnikov Institute of Bioorganic Chemistry, Russian Academy of Sciences, Moscow, Russia.; ${ }^{3}$ Fox Chase Cancer Center, Philadelphia, USA

Background: Cisplatin (Fig. 1) is a low molecular weight platinum-containing compound with a square planar geometry. It is widely used for the treatment of different cancers. Its mode of action is related to its ability to damage DNA through cross-linking of the adjacent purine bases followed by the appearance of different adducts (Fig. 1). The platinum-DNA adducts can induce local distortions of DNA and form micro-loops. Despite the fact that patients initially are responsive to cisplatin therapy, resistance to this drug develops later. The resistance is often associated with overexpression of poly(ADPribose)polymerase 1 (PARP1) in tumor cells. PARP1 acts as a repair enzyme and assists in cell survival after treatment with DNA-damaging agents. Numerous evidence indicate that a level of PARP1 expression and drug-resistance of tumors correlate. The present work is devoted to the study of the effect of cisplatin on PARP1-dependent structural rearrangements of chromatin.
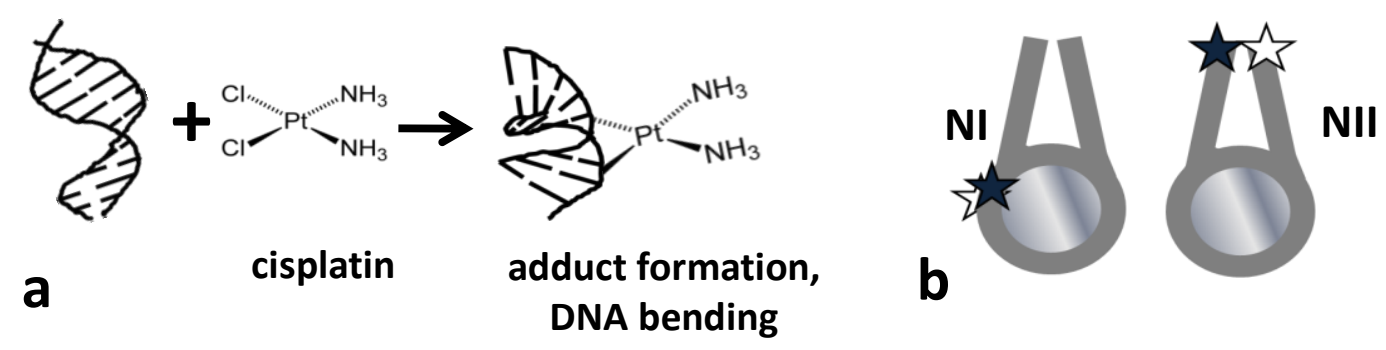

Fig. 1. Experimental system. (a) Cisplatin binds to DNA, bends it and induces adduct formation. (b) The mononucleosomes used in our studies contained 20-bp extending DNA regions. Stars indicate positions of fluorescent Cy3/Cy5 labels. 
Methods: Mononucleosomes were assembled from histone octamers and short DNA containing a nucleosome positioning sequence. To make nucleosomes suitable for structural studies, two single pairs of Cy3/Cy5 labels were introduced into a core or linker region of nucleosomal DNA (NI and NII, respectively, Fig. 1b). Single particle Forster resonance energy transfer microscopy(spFRET-microscopy) was employed to examine structural rearrangements in NI and NII induced by cisplatin and PARP1. Measurements and data analysis were performed as described previously.

Results: spFRET-microscopy analysis of NI and NII treated with CYS10 (10 $\mu \mathrm{M}$ for $12 \mathrm{~h})$ indicates that adduct formation does not affect DNA folding within a core region but changes conformation of DNA in the linker region of a nucleosome. Formation of complexes between PARP1 and nucleosomes affect the DNA structure both in the core and linker regions, increasing the distance between neighboring DNA gyres in the core region and decreasing a distance between DNA linkers. Thus, spFRET-microscopy data show that formation of complexes between PARP1 and nucleosomes pretreated with cisplatin is accompanied by the more pronounced structural rearrangements both in the core and linker regions of nucleosomes as compared to PARP1-nucleosome complexes.

Conclusion: PARP1-induced structural changes in nucleosomes likely facilitate access of the repair enzymes to nucleosomal DNA. The more efficient remodeling activity of PARP1 on cisplatinmodified nucleosomes could explain the more efficient DNA repair of cisplatin-induced lesions and high cisplatin resistance of cancer cells overexpressing PARP1 observed previously.

Key Words: Cisplatin $・$ PARP1・ nucleosomes $・$ spFRET

Sources of Funding: Financial support by RFBR grant №17-00-00163 (17-00-00132, 17-00-00097) is acknowledged.

International Journal of Biomedicine. 2019;9 Suppl 1: S24-25. doi: 10.21103/IJBM.9.Suppl_1.P18

(C)2019 International Medical Research and Development Corporation 